\title{
Las competencias gerenciales en la gestión del cambio de docentes en la unidad de gestión local 01, Perú
}

\author{
The managerial competences in the management of the change of teachers in the local management unit 01, Peru
}

Competências gerenciais em gestão de mudanças de professores na unidade de gestão local 01, Peru

ARTíCULO DE INVESTIGACIÓN

\author{
Myriam Diana Orihuela Alvino \\ myriamorihuela@hotmail.es \\ https://orcid.org/0000-0002-8745-5993 \\ Universidad César Vallejo, Trujillo-Perú \\ Rubén Moisés Mauricio Avalos \\ mauriciodata@gmail.com \\ https://orcid.org/0000-0001-8813-1651 \\ Universidad César Vallejo, Trujillo-Perú
}

\author{
Isabel Menacho Vargas \\ isabelmenachov@gmail.com \\ https://orcid.org/0000-0001-6246-4618 \\ Universidad César Vallejo, Trujillo-Perú \\ José Luis Camarena Mucha \\ arq.jlcamarena@gmail.com \\ https://orcid.org/0000-0003-1099-1701 \\ Universidad César Vallejo, Trujillo-Perú
}

Recibido 24 de mayo 2021 | Arbitrado y aceptado 4 de junio 2021 | Publicado en 01 julio 2021

\section{RESUMEN}

La presente investigación tuvo como objetivo determinar en qué medida las competencias gerenciales influyen en una gestión direccionada al cambio en el personal docente que labora en una unidad de gestión local. Es por esto que a través de cómo los docentes perciben la función del director con objetivo de cambio, se dieron a conocer las fortalezas y debilidades en las competencias gerenciales. Se ha tomado tomado en cuenta una exhaustiva revisión bibliográfica, con respecto a diferentes estudios realizados en torno a las competencias de los directivos de las organizaciones y de cómo estos, enfocan la gestión del cambio en general. El estudio fue de enfoque cuantitativo, con método correlacional. El diseño de investigación fue no experimental y de corte transversal. Para el recolección de datos se utilizó la encuesta a través de dos cuestionarios, siendo la muestra de 156 docentes, quienes laboran en una unidad de gestión local, a partir de la cual se obtuvo como resultado según el pseudo cuadrado Nagelkerke el $100.0 \%$, por lo que se concluye que las competencias gerenciales influyen de manera directa en la gestión del cambio del personal de la institución en estudio.

Palabras clave: Competencias gerenciales; Gestión del cambio; Personal; Unidad de gestión local

\section{ABSTRACT}

The purpose of this research has been to determine to what extent managerial competencies influence management directed to change in the teaching staff working in a local management unit. Thus, through how teachers perceive the role of the director with the objective of change, it allows us to know about the strengths and weaknesses in managerial competencies. From the different studies carried out on the competencies of those who lead organizations and on how change management is approached in general, the relevant information has been taken as the theory on which this research is based. Likewise, the study problem was focused to establish the research problem, the objectives and the hypotheses raised. The approach under which it was carried out is quantitative, the method is correlational, and its design is non-experimental, cross-sectional. For data collection, the survey was used through two questionnaires to a sample of 156 teachers who work in a local management unit, from which $100.0 \%$ was obtained according to the pseudo square Nagelkerke, giving the conclusion that Managerial competencies influence directly the management of personnel change in the institution under study.

Key words: Management competencies; Change management; Personnel; Local management unit

\section{RESUMO}

O objetivo desta pesquisa foi determinar em que medida as competências gerenciais influenciam a gestão voltada para a mudança no corpo docente que trabalha em uma unidade de gestão local. É por isso que, por meio da percepção dos professores sobre o papel do diretor com o objetivo de mudança, foram revelados os pontos fortes e fracos das competências gerenciais. Foi realizada uma revisão bibliográfica exaustiva, no que diz respeito a diferentes estudos realizados sobre as competências dos gestores das organizações e como eles abordam a gestão da mudança em geral. $\mathrm{O}$ estudo teve abordagem quantitativa, com método correlacional. $\mathrm{O}$ desenho da pesquisa foi não experimental e transversal. Para a coleta de dados, utilizouse a survey por meio de dois questionários, a amostra sendo 156 professores, que atuam em uma unidade gestora local, dos quais 100,0\% foi obtido como resultado de acordo com o pseudo-quadrado Nagelkerke, pelo que se conclui que o as competências gerenciais influenciam diretamente na gestão da mudança de pessoal da instituição em estudo.

Palavras-chave: Competências de gestão; Gestão de mudanças; Pessoal; Unidade de gestão local 


\section{INTRODUCCIÓN}

En la sociedad surgen nuevas necesidades, nuevos estilos de vida, aparecen nuevas cosas mientras que el pasado va quedando como experiencias de aprendizaje, las nuevas generaciones se proyectan constantemente hacia el futuro fijando plazos y metas, a este ritmo deben ir las organizaciones en general a través de la gestión del cambio, este es un proceso que las organizaciones modernas consideran necesaria para estar acorde con las exigencias y la globalización, esto involucra a toda la sociedad, por tal razón numerosas empresas han determinado seguir el camino hacia la transformación. Como eje importante en esta gestión de cambio es la persona o grupo humano que tiene a su cargo dirigir los procesos de mejora que contribuyan a la renovación de la estructura de la organización.

Con respecto a las competencias gerenciales que se tienen que tener para realizar esta transformación, cada competencia es un conjunto de capacidades que permiten que el gerente pueda liderar de manera eficaz una organización (Garmendia, 2015). En estas competencias se complementan los conceptos, las habilidades y las actitudes que requiere el gerente en el ejercicio de sus funciones para una exitosa gestión (Castro et al., 2013).

Benavides (2002) realizó una clasificación de las competencias gerenciales diferenciándolas en competencias laborales, competencias genéricas y competencias básicas. Donde las competencias genéricas están estrechamente relacionadas con las características, fines y metas de la organización, mientras que las competencias laborales están referidas al pleno conocimiento de sus funciones como líder de la empresa, por último, las competencias básicas que corresponden al conocimiento elemental, que va desde los primeros años de vida, a la ética y al talento humano.

En ese sentido, Centina et al., (2010) considera que para evaluar el liderazgo de los directivos, es necesario reconocer el carácter social que tiene, considerando la percepción que tiene el grupo liderado, sobre las competencias de su director, por lo que en su investigación, utilizó una escala en las que el grupo tuvo que medir las habilidades directivas de sus jefes, donde rescató que la habilidades, visión compartida y relaciones humanas son buenos predictores para determinar la percepción que tienen los subordinados sobre su jefe.

Con respecto a la gestión del cambio del personal, Martínez (2015) señala como un proceso que implica la toma de estrategias para encaminar al personal para el involucramiento a las etapas de cambio. Por otro lado, para Griffin (2011) indica que la organización entrará a un proceso de estudio en que se evalúe la necesidad de realizar el proceso de cambio; de ser necesario, se inicia la planificación con participación de equipos de trabajo que compromete a los demás trabajadores en los siguientes pasos. Sin embargo, no es una tarea fácil ya que significa lidiar con personas que no están de acuerdo en dejar su zona de confort, o por otras razones, pero al igual, existen múltiples estrategias para contrarrestar este negativismo como son: la sensibilización y los llamados incentivos.

Es necesario aclarar, que, en el área educativa, las instituciones públicas, desde que son creadas deben estar acorde con los propósitos del Estado a través del Ministerio de Educación, por lo tanto, muchas de las reformas, los cambios, los incentivos 
para modificar conductas en el personal docente implica un reajuste de estas normas (Uriarte, 2013). Es por esto que, para llevar a cabo las reformas educativas planteadas, se requiere de la participación de los directivos y docentes afín de poder desarrollar proyectos exitosos y sostenibles en el tiempo, mejorando de esta forma la calidad educativa (Rosas, 2020).

En ese contexto, la calidad que perciben los usuarios con respecto al servicio que brindan las instituciones educativas públicas está considerado como problemática, por lo que (Estrada y Villareal, 2019) resaltan que la formación de directores en México, no es la más adecuada por lo que analizó los procesos de formación de directores de escuelas primarias, identificando las experiencias y desafíos que se encontraron en su formación ; entre ellas se encuentra desafío de ejercer la autoridad en la dinámica de trabajo escolar e involucrar a las familias. Por otro lado, resalta también el desafío de conseguir recursos para implementar acciones para la mejora.

Toribio y Toribio (2017) en su estudio realizado en Perú, elaboraron y valoraron el perfil de las competencias directivas determinantes para los directivos de las instituciones educativas de educación básica, a fin de realizar una mejora continua, se basaron en la normativa internacional. En consecuencia, se obtuvo que las competencias que deben de tener los directivos para gerencial las instituciones educativas son: el manejo de dirección el liderazgo y la gestión del cambio a través de la implementación de programas y actividades que colaboren en alcanzar los objetivos institucionales.

A consecuencia de lo argumentado, esta investigación, se ha enfocado en las instituciones educativas públicas que pertenecen a la una unidad de gestión local 01, cuya atención tiene que satisfacer las necesidades de un gran número de estudiantes y padres de familia, lo que motivó a realizar un estudio desde la efectividad de las competencias gerenciales hacia una gestión del cambio en los docentes. Teniendo en cuenta a Arrascue et al., (2021) quien analizó la percepción de los trabajadores con respecto a las habilidades gerenciales de sus jefes. El estudio tuvo un enfoque cuantitativo, de tipo correlacional y transversal. El cual dio como resultado que las habilidades gerenciales en las dimensiones analíticas e interpersonales fueran consideradas como adecuadas, y las habilidades emocionales, motivación y valores fueran regulares. Además, rescato la variable sociodemográfica de tiempo de servicio, ya que mostró relación con las habilidades gerenciales.

Es por esto que este estudio se ha focalizado en los docentes de la unidad de gestión local, UGEL 01, con la finalidad de determinar el grado de influencia de sus competencias gerenciales en la gestión del cambio del personal docente, quienes laboran en muchos casos durante varios años en un mismo puesto, realizando la misma labor, de manera cómoda y mecánica, esta situación representa un gran reto para los directores, cuyo éxito depende del empleo de estrategias relacionadas a las competencias gerenciales $\mathrm{o}$ directivas para poder avanzar en beneficio de toda la institución educativa, así mismo Griffin (2011) manifiesta que toda empresa para mantenerse debe estar en constante evolución a través del tiempo con cambios de manera periódica y paulatina. 
Por lo tanto, se planteó como hipótesis general, que existe influencia de las competencias gerenciales en la gestión del cambio del personal docente de las instituciones educativas pertenecientes a la UGEL 01. Del mismo modo, como hipótesis específicas se encuentran que las competencias genéricas influyen en la gestión del cambio del personal docente; las competencias laborales influyen en la administración del cambio del personal docente y las competencias básicas influyen en la gestión del cambio del personal docente de las instituciones educativas pertenecientes a la UGEL 01.

\section{MÉTODO}

El método empleado fue el hipotético deductivo, ya que a partir de la revisión de la literatura se enfocó el problema general el cual, posteriormente fue especificado de manera particular en las conclusiones con respecto a la hipótesis (Carrasco, 2009). Tuvo un enfoque cuantitativo, como señalan Hernández, Fernández y Baptista (2014) ya que se efectuó un proceso estructurado para la medición de las variables y obtención de resultados. El tipo de estudio es básico según como lo señala Carrasco (2009) ya que la finalidad es obtener información de la realidad tal como se ha realizado en este estudio. El diseño fue no experimental, transversalcorrelacional, ya que según (Ary, Jacobs y Razavieh, 1982) pretende determinar la relación o grado entre las variables competencias gerenciales y gestión del cambio de docentes. Permitiendo evaluar hasta qué punto las alteraciones de una variable alteraran la otra variable.

Se utilizó como técnica la encuesta, tal como indica, Casas, Repullo y Donado (2003), la cual permite obtener la información de la muestra, la cual estuvo conformada por 156 docentes y el instrumento utilizado fue el cuestionario. Con este criterio se adaptaron dos cuestionarios, el primero para medir las competencias gerenciales del instrumento creado Barba (2011); el tipo de aplicación es directa, de una duración de 10 minutos con característica dicotómica, la cual consta de 30 ítems organizados en tres dimensiones competencias genéricas, competencias laborales y competencias básicas (1-9; 10-19; 20-30). El segundo instrumento mide la gestión del cambio del personal docente, este es un instrumento adaptado de Franco y López (2013); el tipo de aplicación fue directa cuya, de una duración de 10 minutos, de característica politómica, que consta de 16 ítems organizados en dos dimensiones fase de preparación y fase de implantación (1-8; 9-16)

La validez de ambos instrumentos se realizó mediante juicio de expertos, siguiendo los criterios: pertinencia, relevancia y claridad siendo declarado como aplicables. La confiabilidad del instrumento para variable competencias gerenciales se determinó con el coeficiente de KR-20, dando el coeficiente 0.771 . La confiabilidad del instrumento para la variable gestión del cambio del personal se determinó con el coeficiente de alfa de Cronbach, dando el coeficiente 0.938 .

El procedimiento comprendió, en la muestra de 156 docentes, con 4 años o más de servicio para las instituciones educativas públicas pertenecientes a la UGEL 01, de un total de 260 docentes que conformaron la población. Se aplicó el instrumento para medir las competencias gerenciales y otro instrumento para medir la gestión del cambio del personal, en un espacio de 10 min cada uno; la información del primer instrumento fue procesada en un análisis descriptivo de la variable competencias gerenciales; del mismo modo la información del segundo instrumento, la cual fue procesada en un análisis descriptivo de la variable gestión del cambio del personal; los resultados fueron interpretados y a partir de ellos se dieron las conclusiones. 
RESULTADOS otro $48.1 \%$ de docentes mostraron un nivel alto. En Análisis descriptivo de la variable presentó una tendencia de nivel medio.

\section{competencias gerenciales}

En la Tabla 1 se puede apreciar que para el 51,9 $\%$ de los docentes mostraron un nivel medio, y el

Tabla 1. Niveles de la variable competencias gerenciales.

\begin{tabular}{lccc}
\hline & Niveles & f & $\%$ \\
\hline Alto & 75 & 48.1 & \\
Medio & 81 & 51.9 & 0.0 \\
Bajo & 0 & 100.0 \\
Total & 156 & \\
\hline
\end{tabular}

En la Tabla 2 se observa en la dimensión competencias genéricas que para la totalidad de los docentes encuestados mostró un nivel alto. En la dimensión competencias laborales, para el 62,2\% de docentes encuestados mostró un nivel alto y para el 37,8\% de docentes con un nivel medio. En la dimensión competencias básicas para el 59,6\% de docentes encuestados mostró un nivel alto y para el $40.4 \%$ con un nivel medio.

Tabla 2. Niveles de la variable competencias gerenciales por dimensiones.

\begin{tabular}{lcccccc}
\hline \multicolumn{1}{c}{ Niveles } & f & \% & f & \% & f & \% \\
\hline Alto & 156 & 100.0 & 97 & 62.2 & 93 & 59.6 \\
Medio & 0 & 0.0 & 59 & 37.8 & 63 & 40.4 \\
Bajo & 0 & 0.0 & 0 & 0.0 & 0 & 0.0 \\
Total & 156 & 100.0 & 156 & 100.0 & 156 & 100.0 \\
\hline
\end{tabular}

Descripción de la variable gestión del cambio del personal

En la Tabla 3 se observa que para el 23,7\% de docentes encuestados muestran un nivel alto, para el $76.3 \%$ muestran un nivel medio, dando como conclusión que la variable gestión del cambio del personal, muestra una tendencia de nivel medio.

Tabla 3. Niveles de la variable gestión del cambio del personal.

\begin{tabular}{|c|c|c|}
\hline Niveles & $\mathrm{f}$ & $\%$ \\
\hline Alto & 37 & 23.7 \\
\hline Medio & 119 & 76.3 \\
\hline Bajo & 0 & 0.0 \\
\hline Total & 156 & 100.0 \\
\hline
\end{tabular}


En la Tabla 4 se observa que en la dimensión fase de preparación para el $62.2 \%$ de docentes encuestados muestra un nivel alto, para el 37.8\% muestra un nivel medio y para el $0.0 \%$ muestra un nivel bajo. En la dimensión fase de implantación del cambio para el $23.7 \%$ de docentes encuestados muestra un nivel alto, para el $64.1 \%$ muestra un nivel medio y para el $12.2 \%$ muestra un nivel bajo.

Tabla 4. Fase de preparación y fase de implementación.

\begin{tabular}{lcccccc}
\hline \multicolumn{1}{c}{ Niveles } & f & \% & f & \% & f & \% \\
\hline Alto & 97 & 62.2 & 37 & 23.7 & 93 & 59.6 \\
Medio & 59 & 37.8 & 100 & 64.1 & 63 & 40.4 \\
Bajo & 0 & 0.0 & 19 & 12.2 & 0 & 0.0 \\
Total & 156 & 100.0 & 156 & 100.0 & 156 & 100.0 \\
\hline
\end{tabular}

\section{Análisis inferencial}

\section{Prueba de hipótesis general}

$\mathbf{H}_{0}$ : No existe influencia de las competencias gerenciales en la gestión del cambio del personal docente de las instituciones educativas pertenecientes a la UGEL 01 .
$\mathbf{H}_{\mathbf{a}}$ : Existe influencia de las competencias gerenciales en la gestión del cambio del personal docente de las instituciones educativas pertenecientes a la UGEL 01.

Tabla 5. Información de ajuste de los modelos.

\begin{tabular}{lrrrr}
\hline \multicolumn{1}{c}{ Modelo } & $\begin{array}{l}\text { Logaritmo de la } \\
\text { verosimilitud -2 }\end{array}$ & Chi-cuadrado & gl & Sig. \\
\hline Sólo intersección & 170,916 & & 4 &, 000 \\
Final &, 000 & 170,916 & \\
Función de enlace: Logit. & & & \\
\hline
\end{tabular}

En la Tabla 5, para medir la calidad del ajuste del modelo se utiliza los coeficientes Pseudo-R2 de Mc-Fadden, de Cox-Snell y de Nagelkerke. El cálculo de éstos, depende del valor de las de varianzas del modelo final y del modelo inicial con sólo la constante, por lo que fueron calculados de la siguiente manera: Mc-Fadden = 1,000; Cox-Snell = 0,666 y Nagelkerke $=1,000$.

Tabla 6. Pseudo R cuadrado.

\begin{tabular}{ll}
\hline Cox y Snell & 0.666 \\
\hline Nagelkerke & 1.000 \\
Mc Fadden & 1.000 \\
\hline
\end{tabular}

Función de enlace: Logit. 
Del análisis estadístico, en la Tabla 6 se concluye que existe influencia de las competencias gerenciales en la gestión del cambio del personal, de acuerdo al pseudo cuadrado de Nagelkerke en el 100,0\%. A partir de Mc-Fadden = 1,000; Cox-Snell =0,666 y Nagelkerke $=1,000$.

Respecto a las dimensiones de la variable competencias gerenciales, se concluye que las competencias genéricas influyen en la gestión del cambio del personal, de acuerdo al pseudo cuadrado de Nagelkerke en el 37,6\%. Calculado a partir de Mc-Fadden = 0,263; Cox-Snell =0,250 y Nagelkerke $=0,376$. Así mismo, las competencias laborales influyen en la gestión del cambio del personal, de acuerdo al pseudo cuadrado de Nagelkerke en el 52,4\%. Calculado a partir de McFadden $=0,392$; Cox-Snell $=0,524$ y Nagelkerke $=0,524$. De igual forma, las competencias básicas influyen en la gestión del cambio del personal, de acuerdo al pseudo cuadrado de Nagelkerke en el $81,1 \%$. Calculado a partir de Mc-Fadden =0,708; Cox-Snell $=0,540$ y Nagelkerke $=0,811$.

\section{Discusión}

El presente estudio tuvo la finalidad de analizar la variable competencias gerenciales para determinar en qué medida se relaciona con la variable gestión del cambio del personal docente.

Según el resultado obtenido se afirmó que la variable competencias gerenciales en los docentes de las instituciones de la Unidad de Gestión Local 01 , presenta una tendencia de nivel medio. Por otro lado, la variable gestión del cambio del personal en los trabajadores de dicha institución, presenta una tendencia de nivel medio. Por tanto, se tiene como conclusión quelas competencias gerenciales influyen en la gestión del cambio del personal docente de las instituciones educativas pertenecientes a la UGEL 01, de acuerdo al pseudo cuadrado de Nagelkerke en el 100,0\%. En consecuencia, a un determinado nivel de las competencias gerenciales le corresponde el mismo nivel en la gestión del cambio del personal docente. Estos resultados son similares con la investigación efectuada por Nava (2011) en cuya investigación realizada de diseño no experimental, transversal descriptiva, concluyó que las competencias gerenciales son moderadas; además estableció que en la medida que se eleve el nivel de la variable competencias gerenciales, en esa misma medida se elevará el nivel de la segunda variable desempeño laboral en el gerente educativo.

Así mismo Fernández (2015) en un estudio de enfoque cuantitativo y diseño no experimental correlacional, miden la relación entre las competencias gerenciales y productividad laboral en un centro de salud, obteniendo resultados que coinciden con el presente estudio, en cuanto a que existe una relación directa y significativa entre ambas variables luego de haber realizado el análisis de datos tomados a los trabajadores de dicha institución pública con el estadístico de Spearman (sig. Bilateral $=.000<.01 ; \mathrm{Rho}=.546^{\star *}$ ).

Desde otra perspectiva el estudio realizado en una empresa privada por Tito (2012) cuyo propósito fue determinar la relación existente entre las competencias gerenciales y la productividad en empresas de calzado, realizado con un enfoque mixto y en un contexto en que la productividad depende de la demanda de un público exigente, por lo tanto, su política está basada en mantener la calidad y variedad del producto. Los resultados que obtuvo el autor tienen semejanza con los obtenidos en el presente trabajo, en cuanto a que concluye que existe relación entre las competencias gerenciales y la productividad, habiendo quedado demostrada 
su hipótesis, fundamentando de esta manera, la implementación de programas de capacitación a fin de fortalecer de manera sostenida sus competencias gerenciales genéricas, laborales y básicas.

\section{CONCLUSIONES}

Para finalizar se concluye que existe influencia de las competencias gerenciales en la gestión del cambio en el personal docente perteneciente a la UGEL 01. Es decir, si el director de la institución educativa desarrolla sus competencias gerenciales, la gestión del cambio en el personal docente se verá beneficiada. En cuanto a que las competencias genéricas influyen en la gestión del cambio del personal docente que pertenece a la UGEL 01. Es decir, si se desarrolla las competencias genéricas, la gestión del cambio en el personal docente se verá beneficiada. Además, que las competencias laborales influyen en la gestión del cambio del personal docente que pertenece a la UGEL 01. Es decir, si se desarrolla las competencias laborales, la gestión del cambio en el personal docente se verá beneficiada. Y se cierra en que las competencias básicas en la gestión del cambio del personal docente que pertenece a la UGEL 01. Es decir, si se desarrolla las competencias básicas, la gestión del cambio en el personal docente se verá beneficiada.

\section{REFERENCIAS}

Arrascue, I., Podestá, L., Matzumura, J., Gutiérrez, H., y Ruiz, R. (2021). Habilidades gerenciales desde la percepción del personal en el hospital municipal los olivos. 21(2), 275-282. https:// doi.org/10.25176/RFMH.v21i2.3715

Ary, L., Jacobs, Ch. y Razavieh, A. (1982). Introducción a la Investigación Pedagógica (2a Edición). México: Interamericana
Barba, M. (2011). Diagnostico de competencias gerenciales en empresas y universidades de morelos (tesis de doctorado). Universidad Iberoamericana. Mexico

Benavides (2002). Competencias y Competitividad. Diseño para Organizaciones Latinoamericanas. Editorial Mc GRaw Hill. Colombia

Carrasco, S. (2009). Metodologìa de la Investigaciòn Cientifica. Lima: San Marcos

Casas, J., Repullo, C. y Donado, J. (2003). La encuesta como tecnica de investigación, elaboración de cuestionarios y tratamiento estadistico de los datos. Investigación. Obtenido de : file://C:/ Users/user/Desktop/13047738_S300_es.pdf

Castro, L., Fossi, L., Guerrero, W. y Vera, L. (2013). Competencias gerenciales y gestión de los proyectos educativos. Revista Cientifica Ciencias Humanas, 9, 91 - 108. Obtenido de Recuperado de file://G:/Proyecto\%20de\%20 tesis/nuevas\%20dimemnsiones.pdf

Centina, T., Ortega, I., y Aguilar, C. (2010). Habilidades directivas desde la percepcion de los subordinados: Un enfoque relacional para el estudio del liderazgo. 9, 124-137

Estrada, C., y Villareal, A. C. (2019). Desafíos que contribuyen al proceso de formación del director de escuela primaria. 69-86

Fernández, F. (2015). Competencias gerenciales y productividad en el centro materno infantil San Jose - Villa el Salvador, 2015 (tesis de postgrado). Universidad Cesar Vallejo. Lima

Franco, C., y López, L. (2013). Propuesta metodológica para la gestión del cambio cultural aplicable en proyectos de business process management. [Tesis de Maestría, Universidad ICESI]

Garmendia, J. (2015). Competencias Gerenciales de los Empresarios de las Pymis Fronterizas, 
su Realidad Contextual y su Exito. Revista de investigación en administración e ingeniería, 8

Griffin, R. (2011). Comportamiento Organizacional ( $9^{\circ}$ ed.). Mexico: Artgraph.

Hernández, R., Fernández, C. y Baptista, P. (2014). Metodología de la investigación científica. México D. F: Mc Graw-Hill

Martínez, L. (2015). Gestion del cambio y la innovacion en la empresa ( $22^{\circ}$ ed.). Bogota: Ideaspropias

Nava, J. (2011). Competencias gerenciales y el desempeño laboral del gerente educativo en educación media general( tesis de postgrado). Universidad Rafael Urdaneta.Maracaibo, Venezuela

Rosas Hostos, E. F. (2020). Liderazgo pedagógico directivo y desempeño docente en una Institución Educativa de Lima Metropolitana. REVISTA CONCIENCIA EPG, 1(1), 53-63. https://doi.org/10.32654/concienciaepg.1-1.5
Tito, P. (2012). Gestión por competencias y productividad Laboral en empresas del sector confección de calzado de Lima Metropolitana. Universidad Mayor de San Marcos. Lima. Obtenido de : http://cybertesis.unmsm.edu.pe/ bitstream/cybertesis/3155/1/Tito_hp\%282\%29. pdf

Toribio, S., y Toribio, S. (2017). Perfil por competencias gerenciales en directivos de instituciones educativas. 237-252

Uriarte, A. (2013). Instituciòn y Cambio Institucional. En El cambio institucional en la administraciòn pùblica en Mèxico $y$ en Sinaloa (págs. 39-77). Mexico. Obtenido de https://archivos.juridicas.unam.mx/www/bjv/ libros/7/3396/5.pdf 